\title{
Developments in the Role of Clinical Engineers in Blood Purification Therapy
}

\section{Introduction}

Dialysis therapy rapidly gained widespread acceptance from the late 1960s onward in Japan, partly through the support of physicians, nurses, and dialysis technicians working together in team-based medical practice. In the 1970s, the hollow-fiber type dialyzer was developed, and technicians played an important role in the clinical validation, development, and modification of this and other new dialysis-related devices, as well as in the establishment of novel anticoagulation methods. In the 1980s, technicians continued to make substantial contributions to dialysis technology such as computerization and central control systemization. No qualification was required for dialysis technicians at that time. However, in 1987, with technicians now recognized as indispensable professionals in blood purification and in surgeries that used artificial heart-lung machines, clinical engineering was designated an allied medical profession requiring national certification under Japan's Clinical Engineers Act. This made clinical engineers responsible in medical practice for the operation and maintenance of life-support systems such as dialysis machines. Certification permitted them to perform puncture for vascular access (shunt), as well as blood sampling and drug administration via the blood circuit for dialysis. In 1990, the Japan Association for Clinical Engineers (JACE) was founded by qualified clinical engineers. Then, with the government's recognition of its charitable services, JACE became an incorporated association in 2002. This national certification system for clinical engineers is unique to Japan. Clinical engineers are now recognized as being responsible for the operation and management of therapy-related devices in highly advanced and diversified healthcare, as well as being indispensable to safety in healthcare.

\section{The Journey from "Dialysis Technicians" to "Clinical Engineers"}

The history of dialysis technology began in the late 1960s in Japan. The Dialysis Study Group, the predecessor of the Japanese Society for Dialysis Therapy, was established in 1968, and dialysis therapy quickly became widespread after becoming covered by public health insurance in the same year. Physicians started recruiting dialysis technicians specifically for operating and managing dialysis machines, heralding the emergence of an early form of team-based medical care. In the 1970s, the Kiil and Kolff devices were replaced with the hollow-fiber 
dialyzer. Around that time, clinical engineers contributed to the rapid advancements made in several areas of dialysis technology: clinical validation of dialyzers, development of a method for measuring whole blood active coagulation time based on the Lee-White whole blood coagulation time, creation and clinical validation of a filtration-type artificial kidney (hemofiltration machine), and development of a CPU-controlled console. Further contributions were made in the 1980s, with the development and launch of a bicarbonate dialysis fluid supply system and the world's first computerized central dialysis management system. The introduction of online hemodiafiltration in the 1990s prompted the establishment of dialysis fluid purification technology.

It has now been 30 years since Japan's Clinical Engineers Act was enacted. This legislation has provided legal consistency for dialysis technicians and clinical perfusionists, has addressed the problem whereby responsibilities requiring a high degree of expertise were not being completely fulfilled by other medical professionals, and has prepared for the future introduction of innovative technologies in therapies using medical devices.

Table 1 outlines the history of clinical engineering in Japan. Following creation of the Dialysis Technology Network in the Kanto area in 1975, the Exchange Meeting for Dialysis Technicians was established in 1980, and this was later reorganized to become the Japan Association for Dialysis Technicians in 1987 in order to address the development of legal systems. A lawsuit regarding an act of vascular access puncture performed by a dialysis technician in Tochigi Prefecture in 1983 triggered debates in the Diet over the need for national certification of dialysis technicians in 1986, which led to swift enactment of the Clinical Engineers Act in 1987. It took 12 years for this law to be passed, and we have the late Mr. Takeru Shibata, former-President of the Japan Association for Dialysis Technicians, to thank for his leadership during that time.

Newly introduced certification examinations in 1988 produced the first group of clinical engineers, and this was followed 2 years later by the establishment of JACE as a voluntary association. JACE was mainly responsible for conducting scholarly activities to improve the skills of clinical engineers. JACE's subsequent application to the government for incorporated association status, aimed at improving social recognition of the profession, was unfortunately unsuccessful because it coincided with the reform of the public interest corporation system. In the meantime, JACE thought that the anticipated Year 2000 (Y2K) issues would have an impact on the medical field,

Developments in the Role of Clinical Engineers in Blood Purification Therapy so we formulated and published a manual to address that in cooperation with the government, the Japan Medical Association, and the Japanese Nursing Association. This contribution was highly regarded and JACE finally became an incorporated association with public interest corporation status in 2002.

Clinical engineering became well known after the Japanese medical device industry's visions for global competitiveness were published in 2005. Included in this vision were the need to establish medical equipment management rooms and to promote good use of clinical engineers.

After reform of the public interest corporation system, the Cabinet Office became the authorizing body. JACE was evaluated for its public-interest activities and finally became a public interest incorporated association in 2012.

\section{Clinical Engineers and Team-Based Medical Care}

Clinical engineers are medical professionals that have been awarded national certification under the Clinical Engineers Act and work in team-based medical care. For the first time in Japanese medical profession-related law, the responsibility to engage in team-based medical care was set out: Clinical engineers shall work closely with physicians and other medical personnel concerned with ensuring proper medical care.

It is no exaggeration to say that team-based medical care originated from dialysis therapy. In classic 1970s' team-based medical care, the physician, nurse, and dialysis technician were each responsible for their own work and shared tasks at the physician's discretion. Modern medicine has evolved into independent/collaborative team-based medical care where each medical professional is responsible for their own work and cooperates in providing medical care (Fig. 1).

The Clinical Engineers Act defined clinical engineers as professionals who were responsible for the operation, maintenance, and inspection of the life-support systems under a physician's supervision. Thus, the initial interpretation of the law was that clinical engineers are specialists in life-support systems and this interpretation did not include the handling of other medical equipment and devices. However, their position evolved to that of medical device specialists under the Medical Device Industry Visions published in 2005, indicating that the government had come to recognize the role of clinical engineers in the prevention of medical accidents related to medical equipment. Just as pharmacists manage medicines at pharmacies, it was recommended that clinical engineers manage 
Table 1. The 30-year history of clinical engineering in Japan (as of 2018)

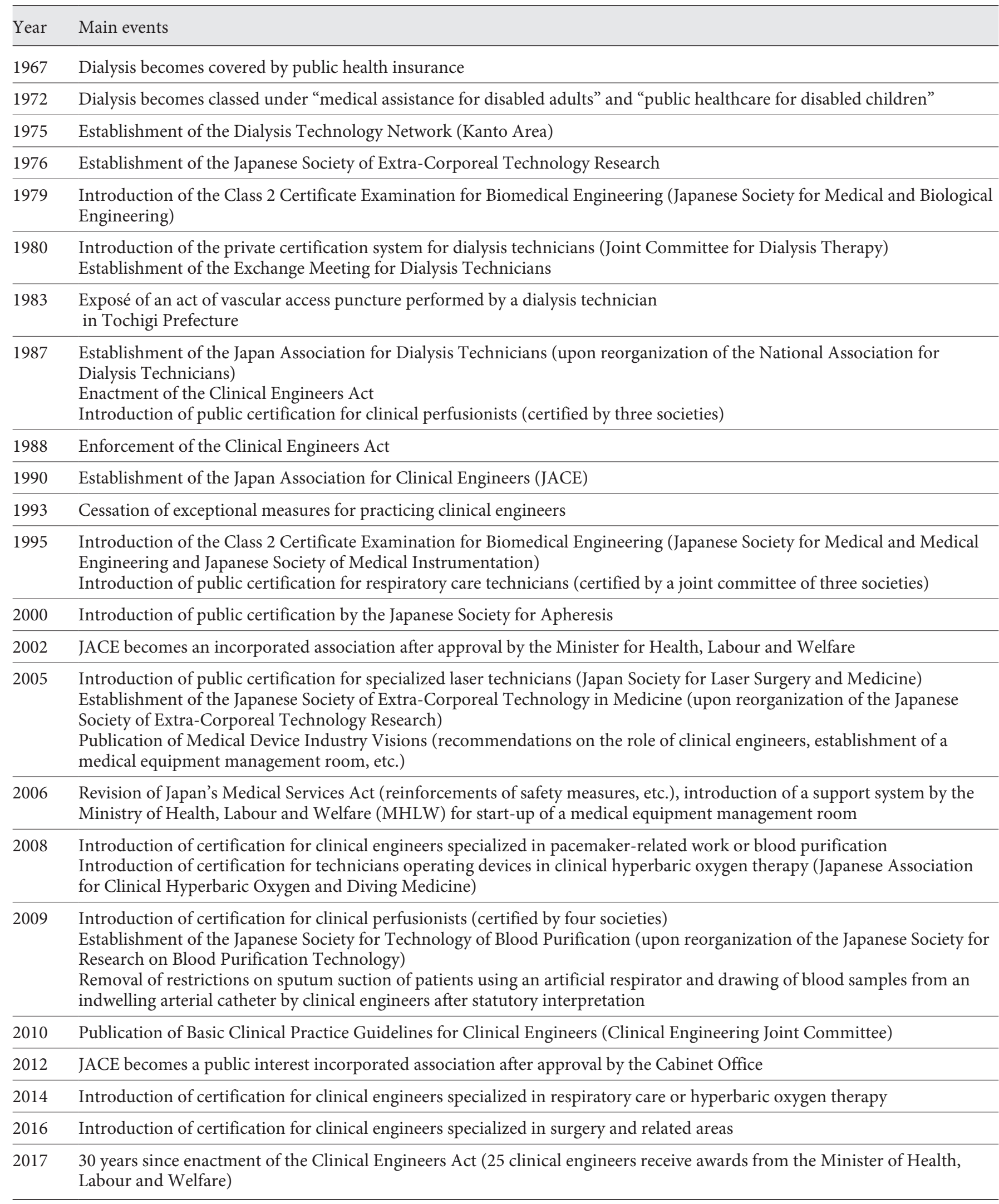


Fig. 1. Introduction of Clinical Engineering and Team-based Medical Care in Japan.

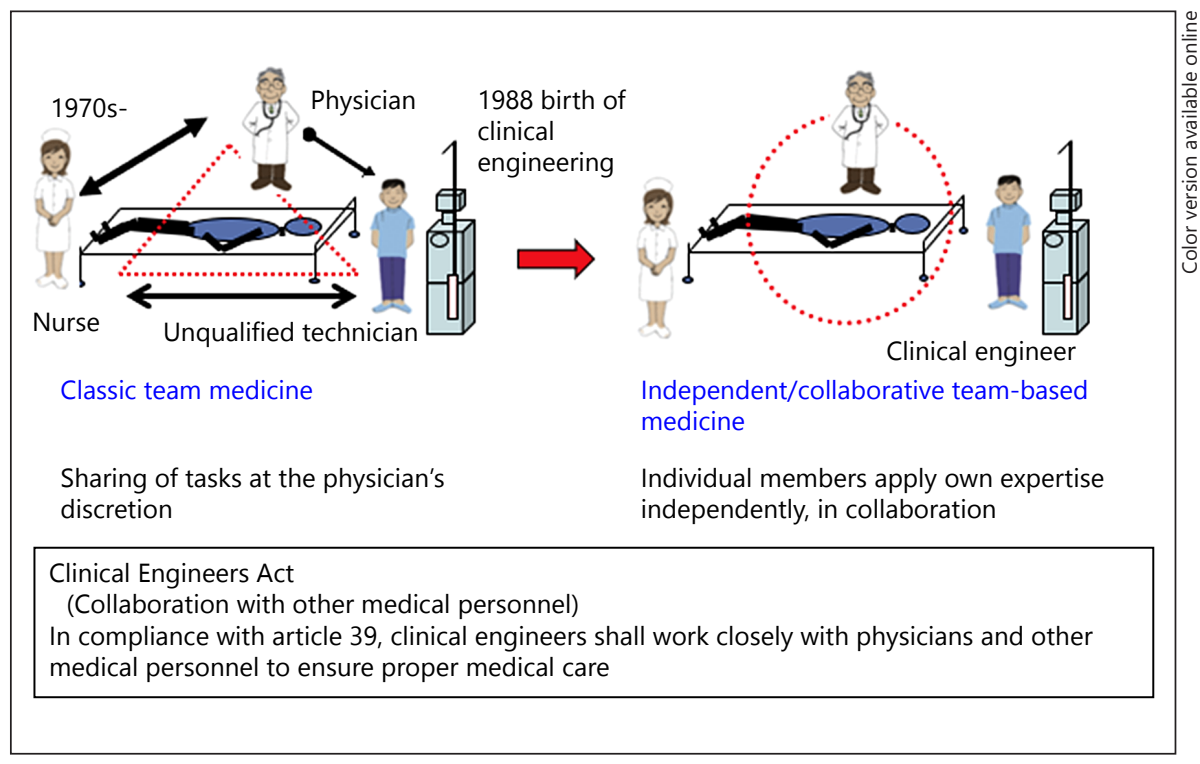

medical equipment in the medical equipment management rooms. As a result, the government introduced a mechanism to pay subsidies to hospitals when they established medical equipment management rooms. Furthermore, a mechanism was introduced whereby hospitals could request medical equipment safety management fees from the government when clinical engineers managed the medical equipment appropriately. Also, dialysis facilities became eligible to receive medical service fees when clinical engineers managed the quality control of dialysis fluids.

These government decisions indicate increasing recognition that medical equipment management is an important component of medical safety and that, consequently, not only the operation of life-support systems but also the management of medical equipment are important responsibilities of clinical engineers.

\section{Clinical Practice Guidelines for Clinical Engineers}

Under the Clinical Practice Guidelines for Clinical Engineers that were issued by the Japanese government in 1988, clinical engineers were not permitted to perform several procedures, such as sputum suction in patients using an artificial respirator or implantable pacemakerrelated procedures. Since around 1993, we had pointed out this issue and requested many times that the government revise the guidelines. However, the government considered it was necessary to revise the law to include such procedures among those permissible for clinical engineers on the grounds that it was not written in law. I had insisted for over 10 years that, because the Clinical Engi-

Developments in the Role of Clinical Engineers in Blood Purification Therapy neers Act does not precisely define procedures that are permissible for clinical engineers, statutory interpretation would likely be enough, with revision of the law not in fact necessary.

In 2007, the guidelines on "Promotion of the distribution of responsibilities among physicians, healthcare workers, and administrative staff" (Table 2) were published to address the issue of overworked physicians, and this provided an opportunity to review the Clinical Practice Guidelines for Clinical Engineers. The government then started discussing "Review meetings for team-based medical care" and the outcomes were reported in March 2010. Finally, from April 30, 2010 after statutory interpretation, in line with what we had argued for many years, sputum suction in patients using an artificial respirator and drawing of blood samples from an indwelling arterial catheter became procedures that can be performed by clinical engineers (Table 3 ).

JACE then formed the "Review Committee for Clinical Practice of Clinical Engineers", comprising 19 related societies and associations and published the "Basic Clinical Practice Guidelines for Clinical Engineers 2010" (see Table 4 for the main points). Following this publication, the "Clinical Practice Guidelines for Clinical Engineers" published in 1988 were abolished.

Statutory interpretation of the clinical practice of clinical engineers takes a negative-list approach. This defines procedures that only physicians can do, does not define the procedures that clinical engineers can do, and does not specify the extent of clinical engineers' responsibilities in medical procedures, such as operating dialysis ma- 
Table 2. Promotion of the distribution of responsibilities among physicians, healthcare workers, and administrative staff (unofficial translation of excerpts)

December 28, 2007, MHLW Health Policy Bureau No. 1228001

Responsibilities should be distributed among healthcare professionals to prevent overwork.

Based on the principal notion of continuous provision of high-quality healthcare services, physicians, nurses, and related professionals should focus on duties that require their expertise so that healthcare services can be effectively provided. ... Management of medical devices: Management (including maintenance) of devices affecting patients' lives and those requiring accurate and complex operation can be performed by clinical engineers under a physician's supervision in accordance with the Clinical Engineers Act. Although, it is also pointed out that physicians and nurses are doing what clinical engineers should do. Reviewing the responsibilities of physicians and nurses, with the active involvement of clinical engineers, ensures safety in healthcare and can reduce the workload of physicians, etc.

Table 3. Promotion of team-based medical care through cooperation and collaboration of healthcare staff (unofficial translation of excerpts)

April 30, 2010, MHLW Health Policy Bureau, No. 0430-1

- Given that sputum suction is requisite for safe and appropriate operation of artificial respirators, this procedure can be considered under "operation of life-support systems" stated in clause 2 of Article 2 of the Clinical Engineers Act and thus can be performed by clinical engineers

- Given that drawing of blood samples from an indwelling arterial catheter is requisite for safe and appropriate operation of artificial respirators, this procedure can be considered under "operation of life-support systems" stated in clause 2 of Article 2 of the Clinical Engineers Act and thus can be performed by clinical engineers

- It is advisable that clinical engineering schools and professional societies review the curriculums and offer training programs, etc. so that clinical engineers can perform the aforementioned procedures safely

Table 4. Main points of the Basic Clinical Practice Guidelines for Clinical Engineers 2010

- Addition of home-based healthcare using a life-support system

- Clear statement about understanding the state of both patients and equipment

- Addition of sputum suction in patients using an artificial respirator and drawing of blood samples from an indwelling arterial catheter

- Recategorization of the "surgical theater and intensive care unit" into "intensive care" and "surgery and related"

- Clear statement about electrosurgical knives, laser and high-energy ultrasound devices, endoscopy devices, and surgical navigation systems in surgery-related devices

- Addition of cardiac and vascular catheterization and implantable defibrillators

chines and performing vascular access puncture. In contrast, legislation for medical technologists uses a positivelist approach, which clearly defines permitted procedures (blood drawing, specimen collection, and 18 items on physiological tests).

It seems then that Japan's Clinical Engineers Act has some flexibility to adopt novel clinical engineering technologies. In fact, the legislation recommends that the clinical practice of clinical engineers be adaptive to changes in healthcare and that appropriate clinical practice corresponding to advances in healthcare technology be ensured through voluntary measures by professional societies and related academic associations.

\section{Responses of Clinical Engineers about Their} Diversified and Demanding Responsibilities

The Basic Clinical Practice Guidelines for Clinical Engineers was published in 2010, and a survey of the actual situation of work revealed that $38.1,15.8$, and $84.1 \%$ of clinical engineers carried out respiratory treatment work, operation of artificial cardiopulmonary machines, and blood purification work respectively, and that they mainly worked in the intensive care unit, where all of these lifesupport systems are integrated, and in the centralized medical equipment management room (Table 5). The results also revealed the rapid spread of arrhythmia therapy, using for example implantable pacemakers, implantable 
Table 5. Distribution of duties of clinical engineers (2012 survey of the actual situation of work)

\begin{tabular}{lrr}
\hline Work area & Number of answers & $\%$ \\
\hline Respiratory treatment work & 1,425 & 38.1 \\
Operation of artificial cardiopulmonary machine & 589 & 15.8 \\
Blood purification work & 3,145 & 84.1 \\
Surgery and related procedures & 808 & 21.6 \\
Intensive care & 789 & 21.1 \\
Cardiac and vascular catheterization & 901 & 24.1 \\
Hyperbaric oxygen therapy & 296 & 7.9 \\
Defibrillator maintenance and management & 1,191 & 31.9 \\
Pacemakers & 811 & 21.7 \\
Implantable defibrillators & 327 & 8.7 \\
Medical equipment maintenance & 2,329 & 62.3 \\
Other & 273 & 7.3 \\
\hline
\end{tabular}

Table 6. Detailed duties in blood purification (2012 survey of the actual situation of work)

\begin{tabular}{|c|c|c|}
\hline Work item & Number of answers & $\%$ \\
\hline $\begin{array}{l}\text { Priming and cleaning of blood circuit for dialysis, determination and alteration of required reagents, } \\
\text { materials, and operation conditions (therapy duration, blood flow, water removal) and monitoring } \\
\text { conditions }\end{array}$ & 3,289 & 98.2 \\
\hline Determination and alteration of volumes of blood and substitution fluids & 2,972 & 88.8 \\
\hline Puncture for vascular access and connection to an indwelling catheter & 3,050 & 91.1 \\
\hline Operation of dialysis machines & 3,279 & 97.9 \\
\hline Measurement and evaluation of the partial pressures of blood gases and coagulation time & 1,960 & 58.5 \\
\hline Time-course monitoring and recording of vital signs & 2,859 & 85.4 \\
\hline Removal of puncture needles and ensuring hemostasis & 3,089 & 92.3 \\
\hline Test data analysis to evaluate dialysis efficiency and dialysis conditions & 2,508 & 74.9 \\
\hline Other & 152 & 4.5 \\
\hline
\end{tabular}

defibrillators, and cardiac catheters, as a new life-support systems area in recent years.

The survey also showed that one clinical engineer was involved in 3.4 work areas; for example, concurrently engaging in dialysis, arrhythmia, and surgery-related work. Knowledge of the latest technologies in other areas is extremely useful in blood purification because it requires the systematic management of patients. Clinical engineers are expected to fulfill this role and, as such, make further contributions to blood purification therapy.

Table 6 shows the duties of clinical engineers in blood purification; the burden of inspection and related duties appears moderate. Procedures with improved safety, such as vascular access puncture using ultrasound diagnostic apparatus, should be used to enhance the quality and safety of healthcare. In addition, clinical engineersas medical device specialists-are expected to fulfill re- 
sponsibilities that involve the operation of advanced surgical machines. Recently, robot-assisted surgery with the use of surgical navigation systems is being performed more frequently, and JACE, in collaboration with the Japan Society for Endoscopic Surgery, is considering establishing an education and training program for specialists in this area.

\section{Conclusion}

In this article, we have described the development of clinical engineering from the emergence of dialysis technicians in the 1970s, through the enactment of the Clinical Engineers Act in 1987, to the latest events in JACE's 30-year history. JACE's mission is "to endeavor to enhance occupational ethics, advance scientific know-how and skills, and improve the reliability of medical and welfare services supported by medical equipment, thereby contributing to healthcare and welfare". JACE is actively conducting projects to achieve this, alongside other activities related to safety measures for the use of medical devices in medical institutions, a topic that is of particular interest to the public. In the field of blood purification specifically, clinical engineers need to pay meticulous attention to ensure safe medical practice given that many dialysis patients need to be managed simultaneously and that diverse complications can occur in elderly patients.

\section{Tadayuki Kawasaki}

Honorary Chairman, Japan Association for Clinical Engineers

Chief Director,

Clinical Engineering Global Promotion Foundation 